\title{
Bosentan Therapy in a Patient with Failed Fontan Procedure: A Case Report
}

\author{
Ting Lu, MD, Mi Tang, MD, Zhong-shi Wu, PhD, Can Huang, MD \\ Department of Cardiovascular Surgery, Second Xiangya Hospital of Central South University, Changsha, Hunan, China
}

\section{ABSTRACT}

Background: Increased pulmonary vascular resistance index (PVR) leads to several complications in patients after a Fontan operation. This increase is mainly attributed to the overexpression of endothelin-1 for a long duration after the Fontan procedure. Here, we describe the case of a 3-yearold boy with a failed Fontan operation who was treated with bosentan, an endothelin-1 receptor blocker.

Case report: Cardiac catheterization was performed, which showed a main pulmonary artery pressure (MPAP) of $19 \mathrm{mmHg}$ and PVRI of 5.6 woods $/ \mathrm{m}^{2}$. Oral bosentan regimen at a dose of $31.25 \mathrm{mg}$ was initiated twice a day. The treatment was continued as pleural effusion and ascites persisted. No adverse events were observed, and the treatment was well tolerated. Pleural effusion disappeared, and ascites decreased markedly after 4 weeks, whereas the MPAP was $15 \mathrm{mmHg}$ and the PVRI was 4.3 woods $/ \mathrm{m}^{2}$. After 3 months of bosentan therapy, the MPAP was $12 \mathrm{mmHg}$ and the PVRI was 4.1 woods $/ \mathrm{m}^{2}$.

Conclusion: We observed that bosentan reduces the PVRI and complications such as pleural effusion and ascites after a failed Fontan procedure.

\section{INTRODUCTION}

The Fontan procedure, which has been used to treat patients with functional univentricular hearts since 1971, has markedly increased survival rates from $50 \%$ to $90 \%$ over the past decades. However, many patients still present with late complications such as arrhythmias, thromboembolizations, protein-losing enteropathy, and lower exercise capacity, which are believed to result from increased pulmonary vascular resistance index (PVRI). Studies have shown that high PVRI may result from either microembolization or pulmonary endothelial dysfunction, especially the overexpression of endothelin-1 after an extended Fontan circulation procedure

Received November 17, 2018; received in revised form fanuary 18, 2019; accepted February 6, 2019.

This study was supported by Hunan Provincial Natural Science Foundation of China (14773037).

Correspondence: Can Huang, PhD, Department of Cardiovascular Surgery, Second Xiangya Hospital of Central South University, \#139 Renmin Road, Changsha, Hunan, 410011, P.R. China; +86-73185292133; fax: +8673185292133 (e-mail: buangcan413@csu.edu.cn).
[Mitchell 2004; Derk 2015]. Therefore, it is believed that the endothelin-1 receptor blocker bosentan is highly effective in patients who have undergone the Fontan procedure. We present the case of a 3-year-old boy who received bosentan therapy after a failed Fontan procedure.

\section{CASE REPORT}

A 3-year-old boy (weight $=12.5 \mathrm{~kg}[27.5 \mathrm{lb}]$ ) with cyanotic lips and clubbing was diagnosed with tricuspid atresia (TA) at birth and subsequently underwent the Glenn shunt procedure when he was one year old. At 3 years of age, cardiac catheterization that was performed before the Fontan procedure showed a main pulmonary artery pressure (MPAP) of $13 \mathrm{mmHg}$ and PVRI of 4.1 woods/m2 (Table). Four devices were used to close three significant systemic collateral pulmonary arteries. Pleural effusion and ascites were observed two weeks after the procedure. Cardiac catheterization showed an MPAP of $19 \mathrm{mmHg}$ and PVRI of 5.6 woods $/ \mathrm{m}^{2}$. Oral bosen$\tan$ regimen at a dose of $31.25 \mathrm{mg}$ was initiated twice a day. Since pleural effusion and ascites (MPAP, $17 \mathrm{mmHg}$; PVRI, 5.6 woods $/ \mathrm{m}^{2}$ ) persisted one month after initiation of therapy, treatment with bosentan was continued. After 4 weeks of therapy, pleural effusion disappeared, and ascites decreased markedly, whereas the MPAP was $15 \mathrm{mmHg}$ and the PVRI was 4.3 woods $/ \mathrm{m}^{2}$. After 3 months of bosentan therapy, the MPAP was $12 \mathrm{mmHg}$ and the PVRI was 4.1 woods $/ \mathrm{m}^{2}$. There were no significant changes in the six-minute walk test (6MWT). Serious hepatic and renal adverse events were not reported.

\section{DISCUSSION}

In 1971, Fontan proposed a new surgical method for treating TA malformations, which involved connecting the superior and inferior vena cava to the pulmonary artery, resulting in a functional single ventricle. As the pulmonary flow is driven without support from the sub-pulmonary ventricle, this procedure was restricted by the PVRI. Bosentan was widely used to reduce the PVRI and the MPAP in patients with pulmonary artery hypertension due to congenital heart defects. However, only few studies on the utilization of bosentan in Fontan patients were reported. Keiichi Hirono showed that bosentan therapy reduced pulmonary artery pressure (PAP) and PVRI in SV patients who did not undergo the Fontan procedure. Since no significant changes in the PVRI and MPAP were observed after 4 weeks of bosentan therapy, 3 and 5 patients in this study underwent the Glenn shunt and 


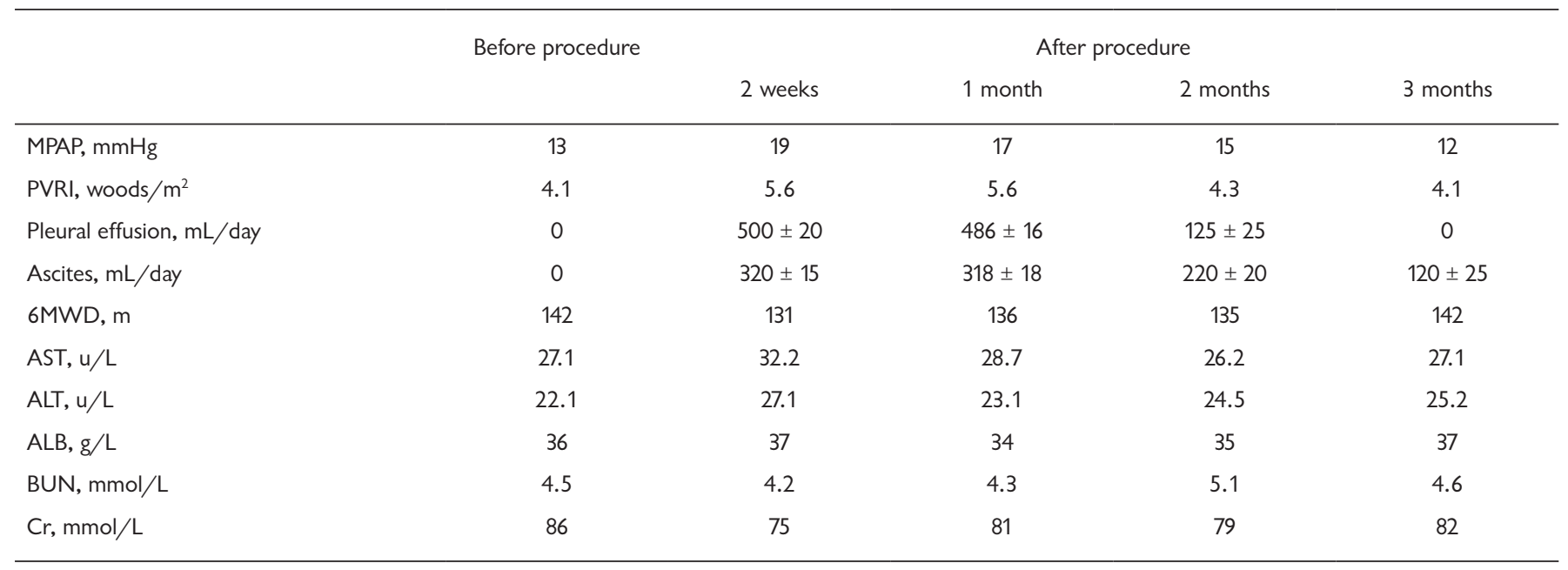

MPAP indicates pulmonary arterial pressure; PVRI, pulmonary vascular resistance; 6MWD, six-minute walking distance; B, bosentan.

Fontan procedures, respectively [Hirono 2010]. Although sildenafil has been previously shown to reduce the PVRI after the Fontan circulation procedure [Goldberg 2011], it was not approved for use in children owing to the dose-dependent effects, which may increase the death rate of pediatric patients with pulmonary artery hypertension. In this case, the boy's PVRI decreased from 5.6 to $4.1 \mathrm{wood} / \mathrm{m}^{2} 4$ weeks after therapy was initiated.

Because of the absence of the sub-pulmonary ventricle, poor cardiac function, pleural effusion, ascites, and protein-losing enteropathy were important early- and late-onset complications. A previous study found that bosentan might increase the cardiac output and the exercise capacity of Fontan patients [Schuuring 2013; Herbert 2014]. In this case, there were no significant changes in the $6 \mathrm{MWD}$ before and after bosentan therapy. Bowater SE suggested that since the 6MWD test has not been validated in Fontan patients, it might not be sensitive enough to measure changes when the baseline value is relatively high [Bowater 2012]. Pleural effusion and ascites were observed in the first 2 weeks. The pleural effusion disappeared, and ascites decreased after 4 weeks, whereas the PVRI and MPAP were reduced after 2 months of bosentan therapy, suggesting the need for a longer treatment duration, which is related to pulmonary hypertension. Since this study was limited to one patient and we did not have a longer follow-up period, we have no reason to believe that bosentan will not effectively improve exercise capacity after the Fontan procedure.

Many drugs are currently used to treat pulmonary hypertension. Bosentan use, which was approved for the treatment of pulmonary artery hypertension in adults, has resulted in possible abnormalities in hepatic and renal function tests. However, there are limited data on children undergoing the Fontan procedure. Bosentan therapy was well tolerated and adverse events were not observed in our patient.
We propose that bosentan therapy reduced the PVRI and occurrence of complications such as pleural effusion, ascites, and heart failure after a failed Fontan procedure. Because of limitations in our patient's activity, the effects of bosentan on exercise capacity and cardiac output need to be confirmed in a future report.

\section{REFERENCES}

Bowater SE, Weaver RA, Thorne SA, Clift PF. 2012. The safety and effects of bosentan in patients with a fontan circulation. Congenit Heart Dis 7:243-9.

Derk G, Houser L, Miner P, et al. 2015. Efficacy of endothelin blockade in adults with fontan physiology. Congenital heart disease. Congenit Heart Dis 10:E11-6.

Goldberg DJ, French B, McBride MG, et al. 2011. Impact of oral sildenafil on exercise performance in children and young adults after the fontan operation: a randomized, double-blind, placebo-controlled, crossover trial. Circulation 123:1185-93.

Hebert A, Mikkelsen UR, Thilen U, et al. 2014. Bosentan improves exercise capacity in adolescents and adults after fontan operation: The TEMPO (Treatment with Endothelin Receptor Antagonist in Fontan Patients, a Randomized, Placebo-Controlled, Double-Blind Study Measuring Peak Oxygen Consumption) Study. Circulation 130:2021-30.

Hirono K, Yoshimura N, Taguchi M, et al. 2010. Bosentan induces clinical and hemodynamic improvement in candidates for right-sided heart bypass surgery. J Thorac Cardiovasc Surg 140:346-51.

Mitchell MB, Campbell DN, Ivy D, et al. 2004. Evidence of pulmonary vascular disease after heart transplantation for Fontan circulation failure. J Thorac Cardiovasc Surg 128:693-702.

Schuuring MJ, Vis JC, van Dijk AP, et al. 2013. Impact of bosentan on exercise capacity in adults after the Fontan procedure: a randomized controlled trial. Eur J Heart Fail 15:690-8. 